\title{
MS-Mittel und Antidiabetika gegen Hirnblutungen
}

\author{
Eine intensive Blutdrucksenkung und Gerinnungs- \\ faktoren in der Akutphase nützen nur wenig, um \\ die Prognose bei einer Hirnblutung zu verbessern. \\ Forscher hoffen jetzt auf schützende Effekte neuer \\ Medikamente.
}

Die Therapie von Hirnblutungen ist bislang keine allzu große Erfolgsgeschichte: Noch immer stirbt etwa jeder dritte Patient an einer solchen Blutung, und viele der Überlebenden kämpfen mit bleibenden Behinderungen. Gefragt sind daher dringend neue und bessere Therapiestrategien.

Ein großes Problem, so Privatdozent Christian Förch, Universität Frankfurt/Main, ist die Hämatomexpansion in der Frühphase der Blutung. Bei rund $40 \%$ kommt es zwischen der dritten und der 20. Stunde nach Blutungsbeginn zu einer deutlichen Ausweitung des Hämatoms, so der Neurologe. Eine Erklärung bietet die „Lawinen-Hypothese“. Platzt eine Hirnarterie, dringt Blut mit hohem Druck in das Hirngewebe ein, dabei wirken hohe Scherkräfte. „Die Zellen platzen in diesem Bereich, es kommt zur Nekrose, und der Zellinhalt wird freigesetzt. Nach einer Stunde ist im Blutungsbereich alles zerstört", sagte Förch. Die Scherkräfte wiederum verursachen auch Rupturen kleinerer Gefäße, und so pflanzt sich die Blutung lawinenartig fort, bis Gerinnungsvorgänge die Oberhand gewinnen. Um eine Hämatomexpansion zu unterbinden, bieten sich daher zwei Möglichkeiten an: den Blutdruck senken und mit Gerinnungsfaktoren die Blutung schneller stillen.

\section{Aggressive Senkung reduziert nicht die Mortalität}

Beide Optionen wurden inzwischen auch ausgiebig getestet, der Nutzen für die Patienten war jedoch geringer. Förch verwies etwa auf die Studie INTERACT II (Intensive Blood Pressure Reduction in Acute Cerebral Haemorrhage Trial), in der über 2.800 Patienten mit intrazerebraler Blutung und einem systolischen Blutdruck zwischen 150 und $220 \mathrm{mmHg}$ entweder zu einer intensiven Blutdrucksenkung (systolischer Wert unter $140 \mathrm{mmHg}$ ) oder zu einer leitlinienbasierten Blutdrucksenkung (systolischer Wert unter $180 \mathrm{mmHg}$ ) eingeteilt wurden. Zwar war die Hämatomexpansion mit der aggressiven Blutdrucksenkung im Schnitt deutlich geringer $(3,1 \mathrm{ml}$ vs. $4,9 \mathrm{ml})$, dies hatte jedoch keinen Einfluss auf die Sterberate nach 90 Tagen (11,9\% vs. 12,0\%). Auch erreichten mit der aggressiven Drucksenkung nur wenig mehr - $48 \%$ versus $44 \%$ - ein gutes Ergebnis (mRS-Wert von 0 -2) auf der modifizierten Rankin-Skala.

Da die Blutung in der Studie mit einem Volumen von im Schnitt $15 \mathrm{ml}$ recht klein war, müssen die Ergebnisse zudem in weiteren Untersuchungen bestätigt werden. Immerhin scheint die aggressive Blutdrucksenkung sicher zu sein und die Prognose nicht zu verschlechtern.

\section{Thrombembolien bei Faktor-VIla-Therapie}

Noch negativer sind die Ergebnisse von Therapieversuchen mit Gerinnungsfaktoren. Förch bezog sich hier auf die FAST-Studie (Factor VIIa in Acute Intracerebral Haemorrhage) aus dem Jahr 2008. Patienten mit Hirnblutungen bekamen darin pro kg/ KG entweder 20 oder $80 \mu \mathrm{g}$ rekombinanten Faktor VIIa oder

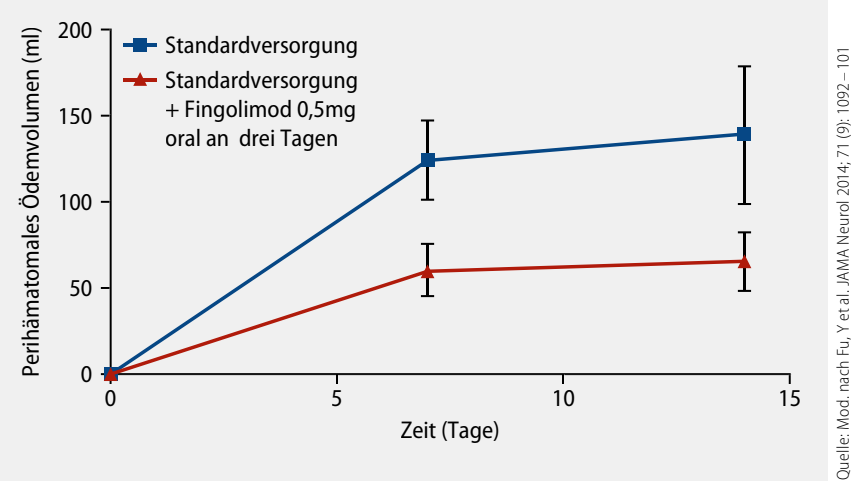

1 Fingolimod zusätzlich zur Standardbehandlung senkt das Volumen des perihämatomalen Ödems signifikant.

Placebo. Tatsächlich wurde die Hämatomausdehnung mit Faktor VIIa im Vergleich zu Placebo gebremst, es gab nach drei Monaten aber praktisch keine Unterschiede bei der Mortalität und dem Behinderungsgrad, dafür häufiger thrombembolische Ereignisse unter der Therapie mit dem Gerinnungsfaktor.

Wenig Nutzen sieht der Neurologe auch für eine frühe Operation bei oberflächlichen Blutungen. Er verwies auf die Ergebnisse der Studie STICH II (Surgical Trial in Lobar Intracerebral Haemorrhage) mit über 600 Patienten. Die frühe Operation konnte weder die Mortalität noch den Behinderungsgrad nach sechs Monaten signifikant senken, allenfalls die Subgruppe der Patienten mit einem Wert auf der Glasgow Coma Scale (GCS) von 9 bis 12 Punkten profitierte. Die Operation bleibt danach wie bisher in den Leitlinien empfohlen eine Option, wenn sich Patienten klinisch verschlechtern.

Etwas breiter scheinen die Eingriffsmöglichkeiten zu sein, um sekundäre Schädigungen zu vermeiden. Als problematisch gilt etwa das bei einer Blutung aus den Erythrozyten freigesetzte Eisen. Es fördert die Bildung freier Radikale und damit die Gewebeschädigung. Auch Entzündungen setzen dem Gewebe nach Ende der Blutung zu. Ermutigend sind hier erste klinische Daten zum anti-inflammatorisch wirkenden MS-Medikament Fingolimod. In einer Proof-of-Concept Studie ließ sich mit Fingolimod zusätzlich zur Standardbehandlung das Volumen des perihämatomalen Ödems deutlich senken ( $\wedge$ Abb. 1).

Auch das Antidiabetikum Pioglitazon gilt als Hoffnungsträger. Es aktiviert Makrophagen und soll Abräumreaktion und damit die Resorption des Hämatoms begünstigen. Das Medikament wird gerade in einer Phase-II-Studie bei Patienten mit Hirnblutungen geprüft. Ebenfalls in einer ersten klinischen Studie untersucht wird der Chelatbildner Deferoxamin. Er könnte das überschüssige Eisen in und um die Läsion herum binden.

Thomas Müller, Springer Medizin

Symposium "Aktuelle Therapiestrategien intrazerebraler Blutungen", 32. Arbeitstagung Neurointensivmedizin (ANIM), Berlin, 30.1.2015 